\title{
High order compact finite difference schemes for a nonlinear Black-Scholes equation
}

\author{
Bertram Düring \\ Fachbereich Mathematik und Statistik \\ Universität Konstanz, Germany \\ Michel Fournié \\ UMR-CNRS 5640, Laboratoire MIP \\ Université Paul Sabatier, Toulouse 3, France \\ Ansgar Jüngel \\ Fachbereich Mathematik und Statistik \\ Universität Konstanz, Germany
}

\begin{abstract}
A nonlinear Black-Scholes equation which models transaction costs arising in the hedging of portfolios is discretized semi-implicitly using high order compact finite difference schemes. In particular, the compact schemes of Rigal are generalized. The numerical results are compared to standard finite difference schemes. It turns out that the compact schemes have very satisfying stability and non-oscillatory properties and are generally more efficient than the considered classical schemes.
\end{abstract}

Keywords. Option pricing, transaction costs, parabolic equations, compact finite difference discretizations.

Acknowledgements. The authors acknowledge partial support from the TMR Project "Asymptotic Methods in Kinetic Theory", grant ERB-FMBXCT97-0157. The first and third author are partly supported by the GerhardHess Program of the Deutsche Forschungsgemeinschaft, grant JU 359/3, and by the AFF Project of the University of Konstanz. The first author acknowledges support from the Center of Finance and Econometrics of the University of Konstanz and expresses his thanks to the MIP Institute of the University P. Sabatier in Toulouse, where a part of this research has been carried out, for the hospitality. 


\section{Introduction}

Financial derivatives, in particular options, became very popular financial contracts in the last few decades. Options can be used, for instance, to hedge assets and portfolios in order to control the risk due to movements in the share price. We recall that a European Call (Put) option provides the right to buy (to sell) a fixed number of assets at the fixed exercise price $E$ at the expiry time $\tau_{0}[10]$.

In an idealized financial market the price of a European option can be obtained as the solution of the celebrated Black-Scholes equation [5, 24]. This equation also provides a hedging portfolio that replicates the contingent claim. However, the Black-Scholes equation has been derived under quite restrictive assumptions (for instance, frictionless, liquid, complete markets). In recent years, nonlinear Black-Scholes equations have been derived in order to model

- transaction costs arising in the hedging of portfolios [1, 6, 9],

- feedback effects due to large traders [12, 13, 14, 16, 26, 32], and

- incomplete markets [22].

The derived time-continuous models are quasi-linear or fully nonlinear parabolic diffusion-convection equations. In this paper we are interested in the option pricing with transaction costs. In 1973, Boyle and Vorst [6] derived from a binomial model an option price that takes into account transaction costs and that is equal to a Black-Scholes price but with a modified volatility of the form

$$
\sigma=\sigma_{0}(1+c A)^{1 / 2}, \quad A=\frac{\mu}{\sigma_{0} \sqrt{\triangle t}}
$$

with $c=1$. Here, $\mu$ is the proportional transaction cost, $\triangle t$ the transaction period, and $\sigma_{0}$ is the original volatility constant. Leland [23] computed the constant $c=(2 / \pi)^{1 / 2}$. Kusuoka [19] then showed that the "optimal" $c$ depends on the risk structure of the market.

Another approach is the maximization of the utility function. For instance, Davis et al. [9] compute the option price as the solution of a nonlinear quasi-variational inequality. This approach has also been used in [1, 15, 37]. It has the disadvantage that the option price depends on the special choice of the utility function. Constantinides and Zariphopoulou [8] obtained universal bounds independent of the utility function.

Parás and Avellaneda [25] derived the modified volatility

$$
\sigma=\sigma_{0}\left(1+A \operatorname{sign}\left(V_{S S}\right)\right)^{1 / 2}
$$


from a binomial model using the algorithm of Bensaid et al. [2]. Here, $V$ is the option price, $S$ the price of the underlying asset, and $V_{S S}$ denotes the second derivative of $V$ with respect to $S$ (the 'Gamma'). In particular, the option price does not need to be convex.

A more complex model has been proposed by Barles and Soner [1]. In their model the nonlinear volatility reads

$$
\sigma=\sigma_{0}\left(1+\Psi\left[\exp \left(\rho\left(\tau_{0}-\tau\right)\right) a^{2} S^{2} V_{S S}\right]\right)
$$

where $\rho$ is the risk-free interest rate, $\tau_{0}$ the maturity, and $a=\mu \sqrt{\gamma N}$, with the risk aversion factor $\gamma$ and the number $N$ of options to be sold. The function $\Psi$ is the solution of the nonlinear initial-value problem

$$
\Psi^{\prime}(A)=\frac{\Psi(A)+1}{2 \sqrt{A \Psi(A)}-A}, A \neq 0, \quad \Psi(0)=0 .
$$

The above model can be derived from a control problem using an exponential utility function in the limit $\varepsilon=1 / \gamma N \rightarrow 0, \mu \rightarrow 0$ such that $a=\mu / \sqrt{\varepsilon}$.

In the mathematical literature, only a few results can be found on the numerical discretization of Black-Scholes equations. The numerical approaches vary from binomial approximations (see, for instance [21] for American options in a stochastic framework), Monte-Carlo methods [20], finite-element discretizations [11, 27], and finite-difference approximations [10]-however, mainly for linear Black-Scholes equations.

The numerical discretization of the Black-Scholes equations with the nonlinear volatilities (1) and (2) has been performed using explicit finite difference schemes [1, 25]. However, explicit schemes have the disadvantage that restrictive conditions on the discretization parameters (for instance, the ratio of the time and space step) are needed to obtain stable, convergent schemes [35]. Moreover, the convergence order is only one in time and two in space.

In this paper we discretize the Black-Scholes equation with nonlinear volatility (2). Our main goal is to obtain efficient and precise schemes, i.e. we wish to derive numerical schemes whose order is superior to standard schemes for second-order equations (like the explicit Euler, the semi-implicit Euler, the Crank-Nicolson and the Leap-Frog Du Fort-Frankel schemes) and whose computing time is comparable to that of classical schemes. Since the equation is of second order, usually a three-point approximation is used [10] and a scheme which is (consistent) of order one in time and two in space is obtained (except the Leap-Frog Du Fort-Frankel scheme; see below). To obtain higher order schemes (of order 2 in time and 4 in space), one possibility is to use more spatial points but this complicates the approximation of the boundary conditions and results in a discretization matrix with larger band 
width. In this paper, we present an alternative using high-order compact schemes which need three points in space only.

More precisely, we study the equation

$$
V_{\tau}+\frac{1}{2} \sigma\left(V_{S S}\right)^{2} S^{2} V_{S S}+\rho S V_{S}-\rho V=0,
$$

where the nonlinear volatility $\sigma\left(V_{S S}\right)$ is given by (2). This equation is solved for the price $S \geq 0$ of the underlying asset and time $\tau_{0} \geq \tau \geq 0$, i.e. backward in time. The terminal condition is

$$
V\left(S, \tau_{0}\right)=V_{0}(S), \quad S \geq 0 .
$$

The equation is derived in [1] for European Call options, i.e. $V_{0}(S)=\max (0, S-$ $E)$, where $E$ is the exercise price. The 'boundary' conditions are as follows:

$$
V(0, \tau)=0, \quad \tau_{0} \geq \tau \geq 0, \quad V(S, \tau) \sim S-E e^{\rho\left(\tau-\tau_{0}\right)} \quad(S \rightarrow \infty) .
$$

The last condition has to be understood in the sense

$$
\lim _{S \rightarrow \infty} \frac{V(S, \tau)}{S-E e^{\rho\left(\tau-\tau_{0}\right)}}=1
$$

uniformly for $\tau_{0} \geq \tau \geq 0$.

We apply the compact schemes R3A and R3B derived by Rigal [29] to the initial-boundary value problem (4)-(6). The nonlinearity is treated explicitly i.e., the final scheme is semi-implicit. The numerical experiments show that, as expected, the $l_{2}$ error is much smaller, for fixed parameters, than the error for the standard schemes (explicit Euler, semi-implicit Euler, Leap-Frog Du Fort-Frankel). The CPU time is only slightly higher for the compact schemes, for a fixed number of grid nodes.

In the nonlinear case, the information on the stability becomes very limited. In any case, linear stability is a necessary condition for the stability of nonlinear problems but it is certainly not sufficient. Particular methods were developed for nonlinear problems. Stetter and Keller studied the nonlinear stability and local stability [18, 34], which have been used successfully for nonlinear ordinary differential equations. Ben-Yu [3] proposed a generalized stability of difference schemes which has been applied widely to numerical solutions of many nonlinear partial differential equations. In this paper we do not use this technique which can be very complicated. Instead, we give results for the linear case $(a=0)$ and validate them in the nonlinear case by numerical studies.

Furthermore, we construct a new three-point compact scheme R3C, which generalizes the scheme R3B of Rigal, and show that this scheme is unconditionally stable and non-oscillatory. The study of the properties of the scheme 
is based upon a thorough Fourier analysis of the Cauchy problem associated with (4). We resort to a local analysis with frozen values of the nonlinear coefficient to make the formulation linear.

Finally, we present a numerical example of a European Call option with different risk aversion constants $a$. As expected, the option price with positive $a$ is higher than the Black-Scholes price $(a=0)$. The difference between the option price for $a>0$ and the Black-Scholes price is maximal (in absolute value) near the strike price $E$, but changes with time. In particular, far from the maturity the difference is maximal at asset prices smaller than the strike price.

The paper is organized as follows. In section 2 the initial-boundary value problem (2)-(6) is reformulated using an exponential transformation. Some classical finite difference schemes are presented and their stability properties are recalled in section 3. A new compact scheme is proposed and analyzed in section 4. Section 5 is devoted to a numerical comparison of the schemes in terms of stability, convergence and efficiency. Finally, in section 6 the solution of the nonlinear Black-Scholes problem is presented for different transaction cost parameters, and some Greeks are computed.

\section{The transformed problem}

In this section we reformulate the problem (4)-(6) using a variable transformation. In [1] the existence of a unique continuous viscosity solution $V$ to this problem has been shown.

To overcome a possible degeneration at $S=0$ and to obtain a forward parabolic problem, we use the variable transformations

$$
x(S)=\ln \left(\frac{S}{E}\right), \quad t(\tau)=\frac{1}{2} \sigma_{0}^{2}\left(\tau_{0}-\tau\right), \quad u=\exp (-x) \frac{V}{E} .
$$

Equation (4) is hereby transformed into

$$
u_{t}-\left(1+\Psi\left[\exp (K t+x) a^{2} E\left(u_{x x}+u_{x}\right)\right]\right)\left(u_{x x}+u_{x}\right)-K u_{x}=0,
$$

with

$$
x \in \mathbb{R}, \quad 0 \leq t \leq T=\sigma_{0}^{2} \tau_{0} / 2, \quad K=\frac{2 \rho}{\sigma_{0}^{2}} .
$$

The problem is completed by the following initial and boundary conditions:

$$
\begin{aligned}
u(x, 0) & =u_{0}(x)=\max (1-\exp (-x), 0), \\
u(x, t) & =0(x \rightarrow-\infty), \\
u(x, t) & \sim 1(x \rightarrow+\infty) .
\end{aligned}
$$




\section{$3 \quad$ Finite Difference Schemes}

In this section we recall standard finite difference schemes and the compact schemes derived by Rigal [29]. For the computation we replace $\mathbb{R}$ by $[-R, R]$ with $R>0$. For simplicity, we consider a uniform grid $Z=\left\{x_{i} \in[-R, R]\right.$ : $\left.x_{i}=i h, i=-N, \ldots, N\right\}$ consisting of $2 N+1$ grid points, with $R=N h$ and with space step $h$ and time step $k$. Let $U_{i}^{n}$ denote the approximate solution of (7) in $x_{i}$ at the time $t_{n}=n k$ and let $U^{n}=\left(U_{i}^{n}\right)_{i=1}^{2 N+1}$. All schemes we consider here use two time levels - except the Leap-Frog Du FortFrankel scheme which uses three time levels. In the space variable $x$ we use a compact stencil requiring only three consecutive points in time level $n+1$. The schemes - except the Leap-Frog Du Fort-Frankel scheme - can be written in the following form:

$$
A^{n} U^{n+1}=B^{n} U^{n}
$$

with the discretization matrices $A^{n}$ and $B^{n}$

$$
A^{n}=\left[a_{-1}, a_{0}, a_{1}\right], \quad B^{n}=\left[b_{-2}, b_{-1}, b_{0}, b_{1}, b_{2}\right] .
$$

The matrix $A^{n}$ is tridiagonal, therefore the resulting linear systems can be solved very efficiently linearly in time using a special form of the Gaussian elimination known as the Thomas algorithm [7]. We suppose that

$$
\sum_{i=-1}^{1} a_{i}=\sum_{i=-2}^{2} b_{i}=1
$$

which is satisfied by any consistent scheme after normalisation of the coefficients.

The nonlinearity is introduced explicitly in all the schemes. In the following let $s_{i}^{n}$ denote the explicitly discretized nonlinear volatility correction:

$$
s_{i}^{n}=\Psi\left[\exp \left(K n k+x_{i}\right) a^{2} E\left(\frac{U_{i-2}^{n}-2 U_{i}^{n}+U_{i+2}^{n}}{4 h^{2}}+\frac{U_{i+1}^{n}-U_{i-1}^{n}}{2 h}\right)\right] .
$$

We use this form of the discretization of the second derivative because it gives a smoother approximation of $u_{x x}$. With a standard central difference the schemes become instable for small values of $h$ unless $k$ is very small. The problem lies in the initial condition $u_{0}$ since it is not differentiable at $x=0$. We use spline interpolation of high order to carefully smooth the initial data, but only in combination with the five-point approximation (9) we obtain acceptable results. 
We use a Dormand-Prince-4-5 Runge-Kutta scheme to solve the ordinary differential equation (3) and a cubic spline interpolation to obtain the values of $\Psi$ for arbitrary arguments (cf. Figure 1).

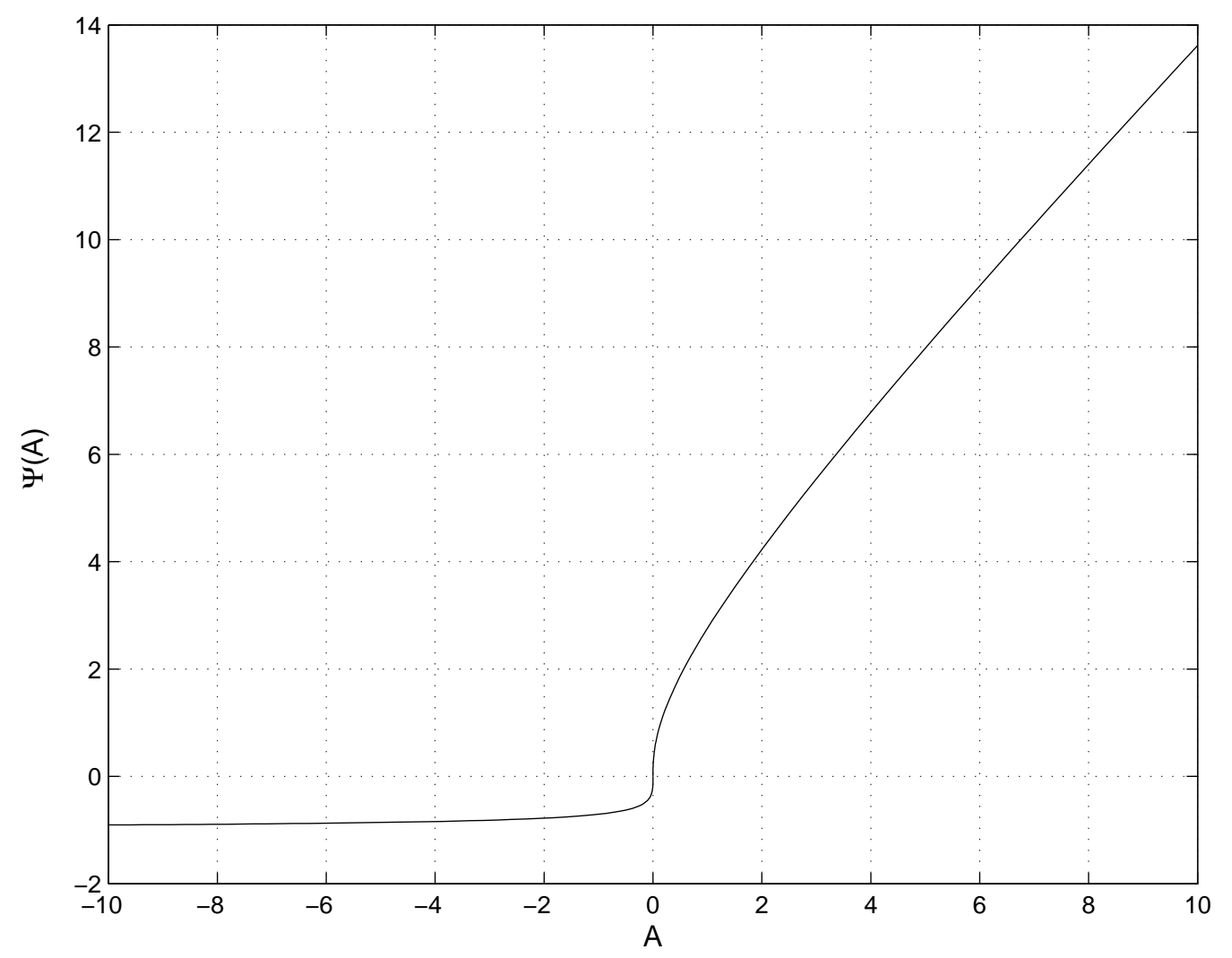

Figure 1: Solution of the ODE (3).

The boundary conditions on the limited grid are treated as follows. Dirichlet conditions are used on both boundary points:

$$
U_{-N}^{n}=0
$$

and

$$
U_{+N}^{n}=1-\exp \left(-x_{N}-K n k\right) .
$$

The latter corresponds to the asymptotic value of the exact solution of the equation for $a=0$. More precisely, the solution of (7) satisfies (see (6)):

$$
u(x, t) \sim 1-\exp (-x-K t) \quad \text { as } x \rightarrow \infty .
$$

Approximately, we have $u(R, t) \approx 1-\exp \left(-x_{N}-K t\right)$ for sufficiently large $R>0$. The nonlinear correction of the volatility in (4) is a function of 
the second derivative, so we assume that the influence of the nonlinearity at the boundary can be neglected for large $R$. The error caused by boundary conditions imposed on an artificial boundary for a class of Black-Scholes equations has been studied rigorously in [17].

In the following let

$$
\lambda=-(1+K), \quad \alpha=\frac{\lambda h}{2}, \quad r=\frac{k}{h^{2}}, \quad \mu=\frac{k}{h}
$$

denote the linear part of the coefficient of the convection term in (7), the so-called cell Reynolds number, the parabolic mesh ratio and the hyperbolic mesh ratio, respectively. We say a scheme is of order $(m, n)$ if it is formally consistent of order $m$ in time and of order $n$ in space or, more precisely, the truncation error is of order $\mathcal{O}\left(k^{m}+h^{n}\right)$.

\subsection{Classical schemes}

In the following we recall some classical finite difference schemes and their properties corresponding to the linear case, i.e. $a=0$ or $s_{i}^{n}=0$. We verify these properties for the nonlinear case $a>0$ by the numerical studies in section 5 .

\subsubsection{Explicit scheme (FTCS)}

The Forward-Time Central-Space explicit scheme (FTCS) is given by

$$
\begin{aligned}
& b_{-2}=\frac{r}{4} s_{i}^{n}, \\
& a_{-1}=0, \quad b_{-1}=r+\frac{\lambda}{2} \mu-\frac{1}{2} \mu s_{i}^{n}, \\
& a_{0}=1, \quad b_{0}=1-2 r-\frac{r}{2} s_{i}^{n}, \\
& a_{1}=0, \quad b_{1}=r-\frac{\lambda}{2} \mu+\frac{1}{2} \mu s_{i}^{n}, \\
& b_{2}=\frac{r}{4} s_{i}^{n}
\end{aligned}
$$

It is of order $(1,2)$, with a very restrictive stability condition. In the linear case it reads [35]:

$$
r \leq \frac{1}{2}
$$

To avoid oscillations, the following condition must be satisfied:

$$
|\alpha| \leq 1
$$




\subsubsection{Semi-implicit scheme (BTCS)}

The semi-implicit scheme, using Backward-Time Central-Space differencing (BTCS) for the linear part and explicit treatment of the nonlinearity, is given by

$$
\begin{array}{rlrl}
b_{-1}=\frac{\lambda}{2} \mu-r, & =\frac{r}{4} s_{i}^{n}, \\
a_{0}=1+2 r, & b_{-1} & =-\frac{1}{2} \mu s_{i}^{n}, \\
b_{0} & =1-\frac{r}{2} s_{i}^{n}, \\
a_{1}=-\frac{\lambda}{2} \mu-r, & b_{1} & =\frac{1}{2} \mu s_{i}^{n}, \\
b_{2} & =\frac{r}{4} s_{i}^{n} .
\end{array}
$$

It is unconditionally stable and of order $(1,2)$. It is non-oscillatory if (12) is satisfied [35].

\subsubsection{Crank-Nicolson (CN)}

The Crank-Nicolson scheme with explicit treatment of the nonlinearity is given by

$$
\begin{aligned}
a_{-1} & =\left(-\frac{r}{2}+\frac{\mu}{4}\right) s_{i}^{n}-\frac{r}{2}-\frac{\lambda}{4} \mu, & b_{-1} & =\left(\frac{r}{2}-\frac{\mu}{4}\right) s_{i}^{n}+\frac{r}{2}+\frac{\lambda}{4} \mu, \\
a_{0} & =1+r\left(1+s_{i}^{n}\right), & b_{0} & =1-r\left(1+s_{i}^{n}\right), \\
a_{1} & =\left(-\frac{r}{2}-\frac{\mu}{4}\right) s_{i}^{n}-\frac{r}{2}+\frac{\lambda}{4} \mu, & b_{1} & =\left(\frac{r}{2}+\frac{\mu}{4}\right) s_{i}^{n}+\frac{r}{2}-\frac{\lambda}{4} \mu
\end{aligned}
$$

and $b_{-2}=b_{2}=0$. It is unconditionally stable and of order $(2,2)$.

\subsubsection{Leap-Frog Du Fort-Frankel scheme (LFDF)}

The Leap-Frog Du Fort-Frankel scheme is an explicit three-time-level scheme. It is given by

$$
\begin{aligned}
\frac{U_{i}^{n+1}-U_{i}^{n-1}}{2 k}= & \frac{U_{i-1}^{n}-\left(U_{i}^{n-1}+U_{i}^{n+1}\right)+U_{i+1}^{n}}{h^{2}}+(1+K) \frac{U_{i+1}^{n}-U_{i-1}^{n}}{2 h} \\
& +s_{i}^{n}\left(\frac{U_{i-2}^{n}-2 U_{i}^{n}+U_{i+2}^{n}}{4 h^{2}}+\frac{U_{i+1}^{n}-U_{i-1}^{n}}{2 h}\right) .
\end{aligned}
$$

Due to the nature of three-time-level schemes we need an additional method to compute the numerical solution at the first time step. In our numerical test in section 5 the compact scheme R3B (see below) is used for this purpose. 
The scheme is stable in the linear case [30] if

$$
r<\frac{1}{2|\alpha|}
$$

and it is of order $(2,2)$. It is non-oscillatory if condition (12) is valid [30].

\subsection{Compact schemes of higher order}

The following two schemes were introduced by Rigal [29] for linear convectiondiffusion problems. We apply them to problem (7). They are both compact two-level schemes of order $(2,4)$ in the linear case. The nonlinearity is treated semi-implicitly as in the previous subsections.

\subsubsection{R3A scheme}

The scheme R3A is given by

$$
\begin{aligned}
a_{-1} & =\left(\frac{1}{12}-\frac{r}{2}\right)(1+\alpha)-\frac{\alpha^{2} r}{6}+\frac{\alpha^{2} r^{2}}{3}, \\
a_{0} & =\frac{5}{6}+r+\frac{\alpha^{2} r}{3}-\frac{2 \alpha^{2} r^{2}}{3}, \\
a_{1} & =\left(\frac{1}{12}-\frac{r}{2}\right)(1-\alpha)-\frac{\alpha^{2} r}{6}+\frac{\alpha^{2} r^{2}}{3}, \\
b_{-2} & =\frac{r}{4} s_{i}^{n}, \\
b_{-1} & =\left(\frac{1}{12}+\frac{r}{2}\right)(1+\alpha)+\frac{\alpha^{2} r}{6}+\frac{\alpha^{2} r^{2}}{3}-\frac{1}{2} \mu s_{i}^{n}, \\
b_{0} & =\frac{5}{6}-r-\frac{\alpha^{2} r}{3}-\frac{2 \alpha^{2} r^{2}}{3}-\frac{r}{2} s_{i}^{n}, \\
b_{1} & =\left(\frac{1}{12}+\frac{r}{2}\right)(1-\alpha)-\frac{\alpha^{2} r}{6}+\frac{\alpha^{2} r^{2}}{3}+\frac{1}{2} \mu s_{i}^{n}, \\
b_{2} & =\frac{r}{4} s_{i}^{n} .
\end{aligned}
$$

This scheme is stable in the linear case $s_{i}^{n}=0[29]$ if

$$
r \leq \frac{1}{\sqrt{2}|\alpha|}
$$

It is non-oscillatory for arbitrary values of $\alpha$. 


\subsubsection{R3B scheme}

The scheme R3B is given by

$$
\begin{aligned}
a_{-1} & =\left(\frac{1}{12}-\frac{r}{2}\right)(1+\alpha)-\frac{\alpha^{2} r}{6}+\frac{\alpha^{3} r^{2}}{3}-\frac{2 \alpha^{4} r^{3}}{3}, \\
a_{0} & =\frac{5}{6}+r+\frac{\alpha^{2} r}{3}+\frac{4 \alpha^{4} r^{3}}{3}, \\
a_{1} & =\left(\frac{1}{12}-\frac{r}{2}\right)(1-\alpha)-\frac{\alpha^{2} r}{6}-\frac{\alpha^{3} r^{2}}{3}-\frac{2 \alpha^{4} r^{3}}{3}, \\
b_{-2} & =\frac{r}{4} s_{i}^{n}, \\
b_{-1} & =\left(\frac{1}{12}+\frac{r}{2}\right)(1+\alpha)+\frac{\alpha^{2} r}{6}+\frac{\alpha^{3} r^{2}}{3}+\frac{2 \alpha^{4} r^{3}}{3}-\left(\frac{r}{4}+\frac{1}{2} \mu\right) s_{i}^{n}, \\
b_{0} & =\frac{5}{6}-r-\frac{\alpha^{2} r}{3}-\frac{4 \alpha^{4} r^{3}}{3}-2 r s_{i}^{n}, \\
b_{1} & =\left(\frac{1}{12}-\frac{r}{2}\right)(1+\alpha)+\frac{\alpha^{2} r}{6}-\frac{\alpha^{3} r^{2}}{3}+\frac{2 \alpha^{4} r^{3}}{3}-\left(\frac{r}{4}-\frac{1}{2} \mu\right) s_{i}^{n}, \\
b_{2} & =\frac{r}{4} s_{i}^{n} .
\end{aligned}
$$

It is unconditionally stable and non-oscillatory in the linear case $s_{i}^{n}=0$ [29].

\section{R3C scheme}

In the nonlinear case $a>0$ it seems to be quite difficult to prove the stability of the schemes presented above. The reason lies in the fact that, using five space points at the present time level, the study of the amplification factor involves certain cubic polynomials. We would need to study their positivity properties for all possible values of the nonlinear coefficients in (7). It turns out that it is not possible to show positivity of these polynomials for arbitrary coefficients.

Therefore we construct now a (semi-implicit) two-level three-point compact scheme which is of high order and can be proved to be stable. We expect that these theoretical benefits will also make the scheme superior in numerical tests. We will use the modified equation technique [36] to construct the scheme.

To obtain an efficient scheme it is important to approximate the nonlinear coefficients in (7) explicitly, i.e. at the time level $n$. Otherwise one would need to perform a nonlinear iteration in each time step which is quite timeconsuming. 
With

$$
\begin{aligned}
& \beta=1+\Psi\left[\exp (K t+x) a^{2} E\left(\Delta_{2} U^{n}+\Delta_{0} U^{n}\right)\right], \\
& \lambda=1+\Psi\left[\exp (K t+x) a^{2} E\left(\Delta_{2} U^{n}+\Delta_{0} U^{n}\right)\right]+K,
\end{aligned}
$$

where

$$
\begin{aligned}
\Delta_{0} U_{j}^{n} & =\frac{U_{j+1}^{n}-U_{j-1}^{n}}{2 h}, \\
\Delta_{2} U_{j}^{n} & =\frac{U_{j+1}^{n}-2 U_{j}^{n}+U_{j-1}^{n}}{h^{2}},
\end{aligned}
$$

the "semi-discretized" equation (7) takes the following form:

$$
u_{t}=\beta u_{x x}-\lambda u_{x} .
$$

We will now study equation (15) for arbitrary values $\beta, \lambda>0$. We define a general two-level three-point scheme

$$
\begin{aligned}
D_{t} U_{j}^{n}= & \beta\left(\frac{1}{2}+A_{1}\right) \Delta_{2} U_{j}^{n}+\beta\left(\frac{1}{2}+A_{2}\right) \Delta_{2} U_{j}^{n+1}-\lambda\left(\frac{1}{2}+B_{1}\right) \Delta_{0} U_{j}^{n} \\
& -\lambda\left(\frac{1}{2}+B_{2}\right) \Delta_{0} U_{j}^{n+1}
\end{aligned}
$$

where

$$
D_{t} U_{j}^{n}=\frac{U_{j}^{n+1}-U_{j}^{n}}{k}
$$

and $A_{i}, B_{i}$ are real constants which must be chosen in such a way that the lower order terms in the truncation error are eliminated and that the scheme is stable and non-oscillatory.

With this explicit discretization of the nonlinear coefficients we study the local stability ('frozen coefficients') of the linearized equations. It is wellknown [28] that for linear problems with variable coefficients (not in general, but for important classes of equations, namely parabolic and symmetric hyperbolic equations) local stability is necessary for overall stability and slightly strengthened local stability is also sufficient to ensure overall stability.

We recall some general results on two-level three-point schemes:

Lemma 1 A two-level three-point finite difference scheme is stable if and only if the coefficients $a_{i}, b_{i}$ satisfy

$$
\begin{aligned}
& \left(a_{1}-a_{-1}\right)^{2}-\left(b_{1}-b_{-1}\right)^{2}>a_{1}+a_{-1}-b_{1}-b_{-1}, \\
& \left(a_{1}+a_{-1}\right)^{2}-\left(b_{1}+b_{-1}\right)^{2}>a_{1}+a_{-1}-b_{1}-b_{-1} .
\end{aligned}
$$


Lemma 2 A two-level three-point finite difference scheme is non-oscillatory if the coefficients $a_{i}, b_{i}$ satisfy

$$
\left(a_{1}-b_{1}\right)\left(a_{-1}-b_{-1}\right) \geq 0 .
$$

For the proofs we refer to [29, Lemmas 1 and 2].

Applying (16) to a sufficiently smooth solution of (15), we obtain the truncation error $E_{u}(k, h)$. Differentiating (15) we obtain higher order equations. Using them to eliminate the time derivatives, we may write $E_{u}(k, h)$ in terms of the space derivatives only:

$$
E_{u}(k, h)=\sum_{j=1}^{4} e_{j} \partial_{x}^{j} u+\text { higher order derivatives }
$$

with

$$
\begin{aligned}
e_{1}= & \lambda\left(B_{2}+B_{1}\right), \\
e_{2}= & -\beta\left(A_{2}+A_{1}\right)-k \lambda^{2} B_{2}, \\
e_{3}= & \frac{1}{12} \lambda\left(2 h^{2}+2 h^{2} B_{2}+6 \lambda^{2} k^{2} B_{2}+k^{2} \lambda^{2}\right. \\
& \left.\quad+12 k \beta A_{2}+2 h^{2} B_{1}+12 k \beta B_{2}\right), \\
e_{4}= & -\frac{1}{24}\left(1+4 B_{2}\right) \lambda^{4} k^{3}-\frac{1}{4}\left(2 A_{2}+4 B_{2}+1\right) \beta \lambda^{2} k^{2} \\
& \quad-\frac{1}{12}\left(\lambda^{2} h^{2}+12 \beta^{2} A_{2}+2 \lambda^{2} h^{2} B_{2}\right) k-\frac{1}{12}\left(1+A_{1}+A_{2}\right) \beta h^{2} .
\end{aligned}
$$

To obtain a scheme of order $(2,4), A_{i}, B_{i}$ must be chosen such that these error terms vanish or are of order $(2,4)$.

Solving the linear system consisting of (20)-(22) in terms of $A_{1}, A_{2}, B_{1}$ we get a class of schemes depending only on the parameter $B_{2}$. This scheme, called (16), is given by (16) with

$$
\begin{aligned}
& B_{1}=-B_{2}, \\
& A_{1}=-\frac{1}{12 k \beta}\left(-2 h^{2}+6 \lambda^{2} k^{2} B_{2}-k^{2} \lambda^{2}-12 k \beta B_{2}\right), \\
& A_{2}=-\frac{1}{12 k \beta}\left(2 h^{2}+6 \lambda^{2} k^{2} B_{2}+k^{2} \lambda^{2}+12 k \beta B_{2}\right) .
\end{aligned}
$$

Equation (24) is a necessary condition to have a consistent scheme. The coefficient $B_{2}$ should be chosen in such a way that we obtain a stable and non-oscillatory scheme of order $(2,4)$. Further we require our scheme to be forward diffusive (in relation with the parabolic problem being well-posed), i.e.

$$
1+\beta A_{1}+\beta A_{2}>0 .
$$

We now study the properties of the scheme (16). We obtain the following result: 
Theorem 3 Scheme (16') is stable if and only if the coefficient $B_{2}$ and $r, \alpha, \beta$ satisfy

$$
\left(-\beta+4 r \alpha^{2} B_{2}\right)\left(-1+4 r^{2} \alpha^{2}+12 \beta r B_{2}\right)>0 .
$$

It is non-oscillatory if $B_{2}$ and $r, \alpha, \beta$ satisfy

$$
\left(-\beta+4 r \alpha^{2} B_{2}+\alpha\right)\left(-\beta+4 r \alpha^{2} B_{2}-\alpha\right) \geq 0 .
$$

It is forward diffusive if and only if $B_{2}, r, \alpha$ satisfy

$$
1-4 r \alpha^{2} B_{2}>0 \text {. }
$$

Proof. To prove stability we need to verify conditions (17) and (18). This is shown by straightforward computation. The scheme (16) can be written in the form (8) where the coefficients $a_{i}, b_{i}$ are given by

$$
\begin{aligned}
a_{-1} & =-\beta\left(\frac{r}{2}+r A_{2}\right)-\frac{\mu}{4}-\mu \frac{B_{2}}{2}, & b_{-1} & =\beta\left(\frac{r}{2}+r A_{1}\right)+\frac{\mu}{4}+\mu \frac{B_{1}}{2}, \\
a_{0} & =1+\beta\left(r+2 r A_{2}\right), & b_{0} & =1-\beta\left(r+2 r A_{1}\right), \\
a_{1} & =-\beta\left(\frac{r}{2}+r A_{2}\right)+\frac{\mu}{4}+\mu \frac{B_{2}}{2}, & b_{1} & =\beta\left(\frac{r}{2}+r A_{1}\right)-\frac{\mu}{4}-\mu \frac{B_{1}}{2}
\end{aligned}
$$

and $b_{-2}=b_{2}=0$. With these coefficients (17) and (18) are equivalent to

$$
\begin{aligned}
\left(B_{2}+B_{2}^{2}-B_{1}-B_{1}^{2}\right) \mu^{2}+\left(2 \beta+2 \beta A_{2}+2 \beta A_{1}\right) r & >0, \\
-2 r \beta\left(1+A_{1}+A_{2}\right)\left(2 \beta r A_{1}-2 \beta r A_{2}-1\right) & >0 .
\end{aligned}
$$

Using (24)-(26) these inequalities simplify to

$$
\begin{aligned}
2 \beta r & >0, \\
\frac{2}{3} r\left(-\beta+4 r \alpha^{2} B_{2}\right)\left(-1+4 r^{2} \alpha^{2}+12 \beta r B_{2}\right) & >0,
\end{aligned}
$$

respectively. Condition (31) is always satisfied and (32) yields (28).

For non-oscillation we have to check condition (19). Elementary computations using the above coefficients and substituting $B_{1}, A_{1}, A_{2}$ from (24)-(26) give the condition

$$
\frac{1}{4}\left(-2 r \beta+2 r k \lambda^{2} B_{2}+\mu\right)\left(-2 r \beta+2 r k \lambda^{2} B_{2}-\mu\right) \geq 0 .
$$

Writing this condition in terms of $\alpha$ and $r$ gives (29).

Substituting (25) and (26) into (27) we obtain

$$
1-k \lambda^{2} B_{2}>0
$$


which is equivalent to (30).

We will now propose a choice of the coefficient $B_{2}$ and study the properties of the scheme obtained by this choice. By construction, $e_{1}=e_{2}=e_{3}=0$ for the scheme (16). The error $e_{4}$ can be written as:

$$
e_{4}=\frac{1}{12} k\left(-\lambda^{2} h^{2}+k^{2} \lambda^{4}+12 \beta^{2}\right) B_{2}-\frac{1}{12} \beta\left(-h^{2}+2 k^{2} \lambda^{2}\right) .
$$

We must choose $B_{2}$ in such a way that $e_{4}$ is of order $(2,4)$. The lower order part of $e_{4}$ is

$$
k \beta^{2} B_{2}+\frac{1}{12} \beta h^{2}
$$

Therefore we make the ansatz

$$
B_{2}=-\frac{1}{12} \frac{h^{2}}{\beta k}+b
$$

We have to choose the constant $b$ of order $\mathcal{O}\left(h^{4}\right)$ to obtain a truncation error $e_{4}$ of the same order. The obvious choice $b=0$ is not recommended since in the linear case $\beta=1$ or $a=0$, this choice leads to the R3A scheme which is not unconditionally stable. We want to choose $b$ in such a way that the conditions (28)-(30) of Theorem 3 are satisfied.

We define the R3C scheme by choosing

$$
b=-\frac{r \alpha^{2}}{3 \beta}=-\frac{\lambda^{2} k}{12 \beta}
$$

which yields

$$
B_{2}=-\frac{1+4 r^{2} \alpha^{2}}{12 \beta r}
$$

For the (linear) case $\beta=1$ this choice corresponds to the R3B scheme of 
Rigal [29]. The coefficients $a_{i}, b_{i}$ are given by:

$$
\begin{aligned}
a_{-1} & =-\frac{12 r \beta^{2}-2 \beta+r \lambda^{2} h^{2}+r^{3} \lambda^{4} h^{4}+6 r \lambda h \beta-\lambda h-r^{2} \lambda^{3} h^{3}}{24 \beta}, \\
a_{0} & =\frac{10 \beta+12 r \beta^{2}+r \lambda^{2} h^{2}+r^{3} \lambda^{4} h^{4}}{12 \beta}, \\
a_{1} & =-\frac{12 r \beta^{2}-2 \beta+r \lambda^{2} h^{2}+r^{3} \lambda^{4} h^{4}-6 r \lambda h \beta+\lambda h+r^{2} \lambda^{3} h^{3}}{24 \beta}, \\
b_{-1} & =\frac{12 r \beta^{2}+2 \beta+r \lambda^{2} h^{2}+r^{3} \lambda^{4} h^{4}+6 r \lambda h \beta+\lambda h+r^{2} \lambda^{3} h^{3}}{24 \beta}, \\
b_{0} & =-\frac{-10 \beta+12 r \beta^{2}+r \lambda^{2} h^{2}+r^{3} \lambda^{4} h^{4}}{12 \beta}, \\
b_{1} & =\frac{12 r \beta^{2}+2 \beta+r \lambda^{2} h^{2}+r^{3} \lambda^{4} h^{4}-6 r \lambda h \beta-\lambda h-r^{2} \lambda^{3} h^{3}}{24 \beta} .
\end{aligned}
$$

Theorem 4 The R3C scheme defined above is an unconditionally stable, non-oscillatory and forward diffusive scheme of order (2,4). Its truncation error is given by

$$
e_{4}=-\frac{\lambda^{2}\left(k^{4} \lambda^{4}-h^{4}+36 k^{2} \beta^{2}\right)}{144 \beta} .
$$

Proof. Substituting (35) in (33) we get (36), i.e. the scheme is of order $(2,4)$, since $e_{1}=e_{2}=e_{3}=0$.

Taking into account (35), conditions (30) and (28) are equivalent to

$$
\begin{aligned}
1+\frac{\alpha^{2}\left(1+4 \alpha^{2} r^{2}\right)}{3 \beta} & >0, \\
\frac{4}{3}\left(\beta+\frac{\alpha^{2}\left(1+4 \alpha^{2} r^{2}\right)}{3 \beta}\right) & >0,
\end{aligned}
$$

respectively. These conditions hold for all values of $\beta$.

Using (35) in (29), we find that this condition is valid if for all values of $\beta$

$$
\frac{1}{9} \frac{r^{2}}{\beta^{2}}\left(3 \beta^{2}+\alpha^{2}+4 \alpha^{4} r^{2}+3 \beta \alpha\right)\left(3 \beta^{2}+\alpha^{2}+4 \alpha^{4} r^{2}-3 \beta \alpha\right) \geq 0
$$

or, equivalently, if for all values of $\beta$

$$
3 \beta^{2}-3 \alpha \beta+\alpha^{2}+4 \alpha^{4} r^{2} \geq 0 .
$$

This is a quadratic polynomial in $\beta$. Its leading coefficient is positive and its discriminant is equal to $-3 \alpha^{2}-48 \alpha^{4} r^{2}$ which is negative for all values of $\alpha$ and $r$. Hence there are no roots and (38) is true for any value of $\beta$. 
Remark 5 In the linear case $\beta=1$ or $a=0$ the R3C scheme coincides with the R3B scheme.

\section{$5 \quad$ Numerical study}

\subsection{Stability}

To study the stability of the schemes numerically, we compute the $l_{2}$-error $\varepsilon_{2}$ of the numerical solutions for different values of $\alpha$ and $r$, where

$$
\varepsilon_{2}=\left(h \sum_{i=-N}^{+N}\left|U_{i}^{k_{T}}-u\left(x_{i}, T\right)\right|^{2}\right)^{\frac{1}{2}}
$$

and $T=k_{T} k$. In the linear case $a=0$ we use the exact solution and in the nonlinear case $a>0$ a solution on a very fine grid (with $N=800$ ) as reference solution. The computations were done using the following parameters

$$
\sigma_{0}=0.45, \quad \rho=0.1, \quad E=100, \quad T=0.50625 .
$$

In Figure 2 the error $\varepsilon_{2}$ is plotted in the $\alpha-r$-plane (see (10) for each classical scheme and in Figure 3 for the compact schemes for the linear case $(a=0)$ and the nonlinear case $(a=0.02)$. Different scales were used for the error of classic and compact schemes. We notice that the schemes' behaviour is similar in both cases:

- FTCS: The conditions (11) and (12) for the stability and non-oscillation can be found again numerically. For large values of $\alpha$ and $r$, the scheme is unstable and oscillations occur. The area in which the scheme produces acceptable results is very small. In the nonlinear case the stability area is even smaller.

- BTCS: The $l_{2}$-error of this scheme is large for larger values of $\alpha$, giving unsatisfactory results for this region (oscillations).

- LFDF: For large values of $\alpha$ the error grows rapidly. The errors are slightly smaller than those of the BTCS scheme.

- CN: The error in the linear case is small compared to the other classical schemes. In the nonlinear case the error grows fast for large values of $\alpha$.

- R3A: The error of this scheme shows its good properties. No oscillations occur, the stability region is very large as predicted by (14). 
- R3B: We observe that scheme R3B gives a slightly better behavior than R3A. There are no oscillations and the scheme is unconditionally stable.

- R3C: As predicted by our theoretical results the scheme is unconditionally stable and non-oscillatory. In the nonlinear case $(a>0)$ the error is even smaller than that of R3A/R3B. In the linear case $(a=0)$ the result is identical to that of $\mathrm{R} 3 \mathrm{~B}$ (cf. Remark 5).
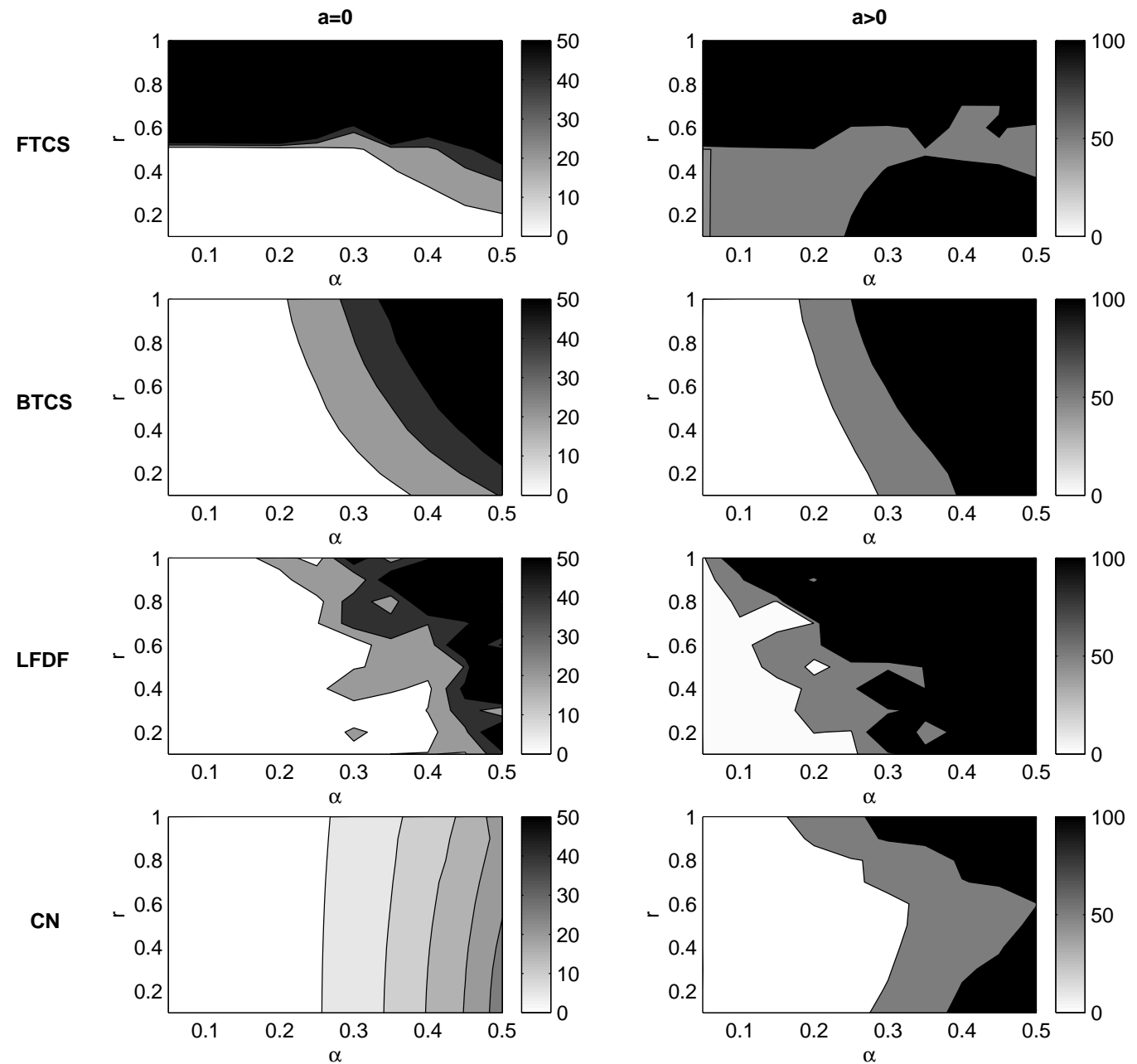

Figure 2: Classical schemes: $l_{2}$-error in the $r-\alpha$-plane.

Comparing the computational results of the different schemes we can make the following observations. In the small region of the $\alpha-r$-plane, where the FTCS scheme is stable, the error of all classical schemes is about 

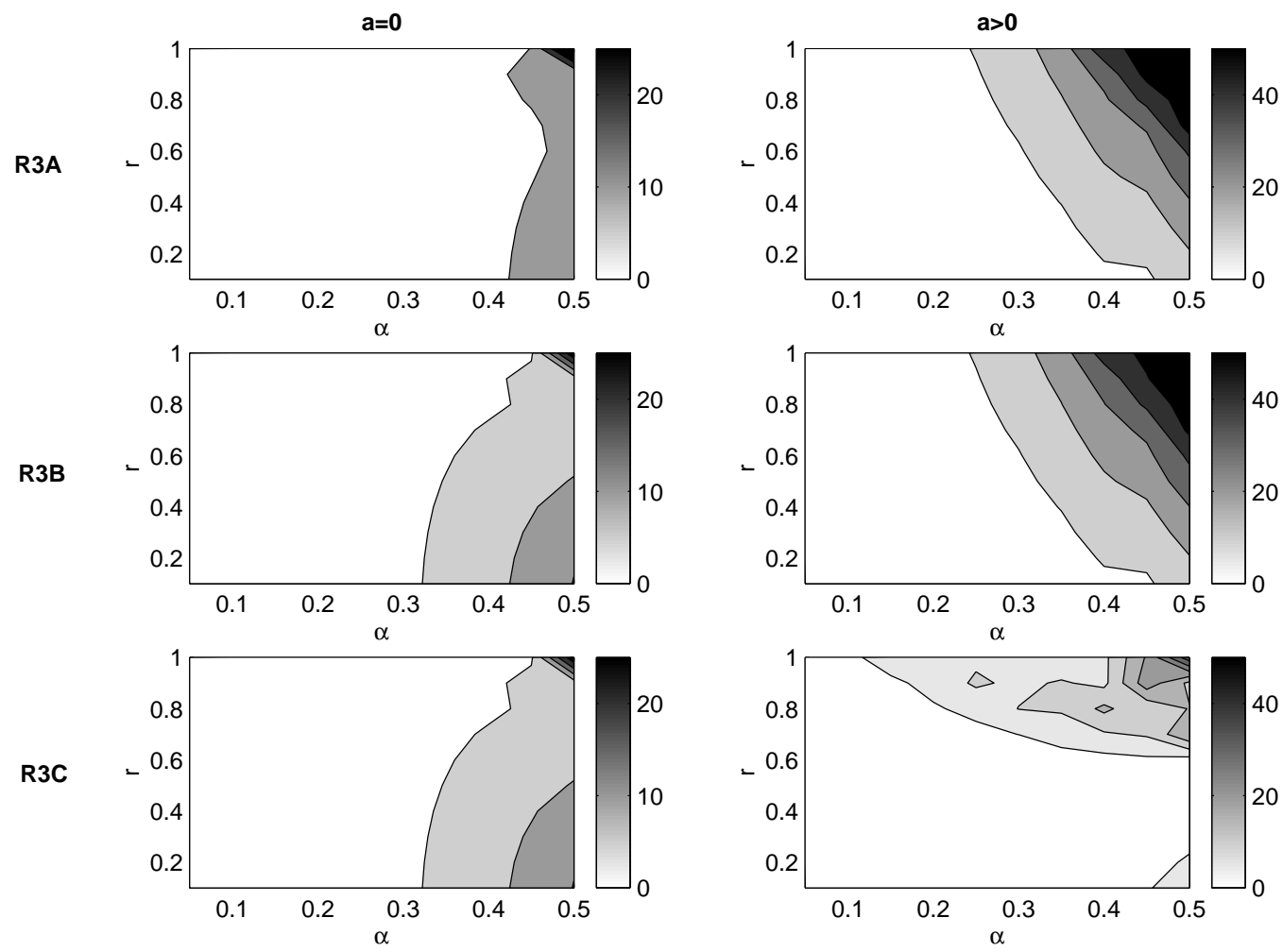

Figure 3: Compact schemes: $l_{2}$-error in the $r-\alpha$-plane.

the same. The CN scheme gives the best results of all the classical schemes. Comparing the classical (FTCS, BTCS, LFDF, CN) to the high order compact schemes, we notice the superiority of the compact schemes. They are generally significantly more accurate than the classical schemes (due to their higher order); they show no oscillations and their use is not restricted by strong stability conditions. The error difference between R3A and R3B is insignificant whereas $\mathrm{R} 3 \mathrm{C}$ provides even better results in the nonlinear case.

\subsection{Convergence}

The truncation error given by expression (36) represents the pointwise error in approximating the differential equation (but not necessarily the solution) [35]. We present in this section a numerical study to compute the order of convergence of the R3C scheme. Asymptotically, we expect the pointwise error to converge as

$$
\varepsilon_{2}=C h^{m}
$$




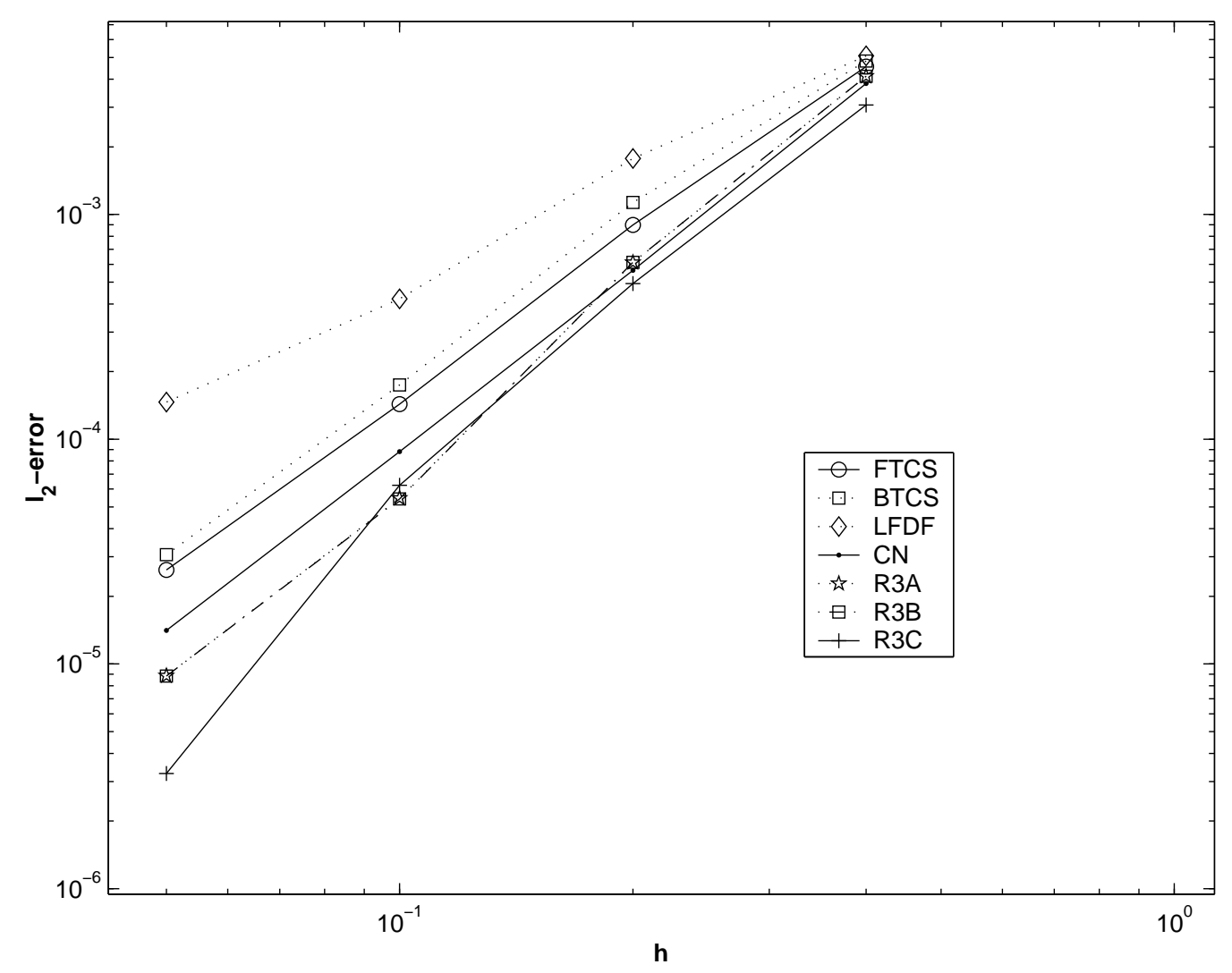

Figure 4: Numerical Convergence: $l_{2}$-error vs. $h$.

for some $m$ and $C$ representing a constant. This implies

$$
\log \left(\varepsilon_{2}\right)=\log (C)+m \log (h) .
$$

Hence, the double-logarithmic plot $\varepsilon_{2}$ against $h$ should be asymptotic to a straight line with slope $m$. This gives a method for experimentally determining the order of accuracy of the method. We refer to Figure 4 for the results with the parameters

$$
a=0.02, \quad \sigma_{0}=0.45, \quad \rho=0.1, \quad E=100, \quad T=0.009375 .
$$

Table 1 summarizes the maximal, minimal and average numerical convergence rates. We observe that the numerical convergence rates roughly correspond to the order of the schemes.

\subsection{Efficiency}

An important point in our comparison is the efficiency of the schemes, i.e. the computation time to obtain a given accuracy. Obviously this is machine 


\begin{tabular}{|c||c|c||c|}
\hline & $m_{\max }$ & $m_{\min }$ & $m_{a v}$ \\
\hline \hline FTCS & 2.65 & 2.34 & 2.48 \\
\hline BTCS & 2.70 & 2.09 & 2.43 \\
\hline LFDF & 2.08 & 1.52 & 1.80 \\
\hline CN & 2.76 & 2.64 & 2.69 \\
\hline R3A & 3.41 & 2.75 & 3.13 \\
\hline R3B & 3.42 & 2.75 & 3.13 \\
\hline R3C & 4.26 & 2.98 & 3.29 \\
\hline
\end{tabular}

Table 1: Convergence rates.

as well as programming dependent. The different schemes were implemented in an efficient and consistent manner in order not to bias any of them. The computation times recorded include the time for matrix setups, inversions and boundary condition evaluation. All results were computed on the same machine. The number of operations to solve the tridiagonal systems with the Thomas Algorithm is of order $\mathcal{O}(N)$ (see section 3 for the definition of $N)$. Hence the dominant factor in the running time is the matrix setup, not the inversion.

We computed solutions on grids with $N=10,20,30,40$. In Figure 5 we plotted the relative $l_{2}$-error versus the CPU time for the different grids and schemes. We see that for fixed error the compact schemes take less CPU time than the classic schemes. Due to the strong stability condition of the FTCS scheme, it is generally very time consuming. For fixed time the error of the compact schemes is always significantly smaller. The three compact schemes are the most efficient ones where the R3C scheme seems to be superior to the R3A and R3B schemes.

Using the same values of $\alpha$ and $r$, the implicit schemes' (BTCS, CN, R3A, R3B, R3C) computation time is not much larger than that of the explicit schemes (FTCS, LFDF), but the accuracy of the compact schemes is significantly better.

\section{Financial Example}

The Black-Scholes analysis requires continuous trading of the hedged portfolio and this may be expensive in a market with proportional transaction costs. To show the influence of the transaction costs on the price of the European Call option, we compute the price given by the numerical solution of (4) and the standard Black-Scholes value for the following choice of parameters 


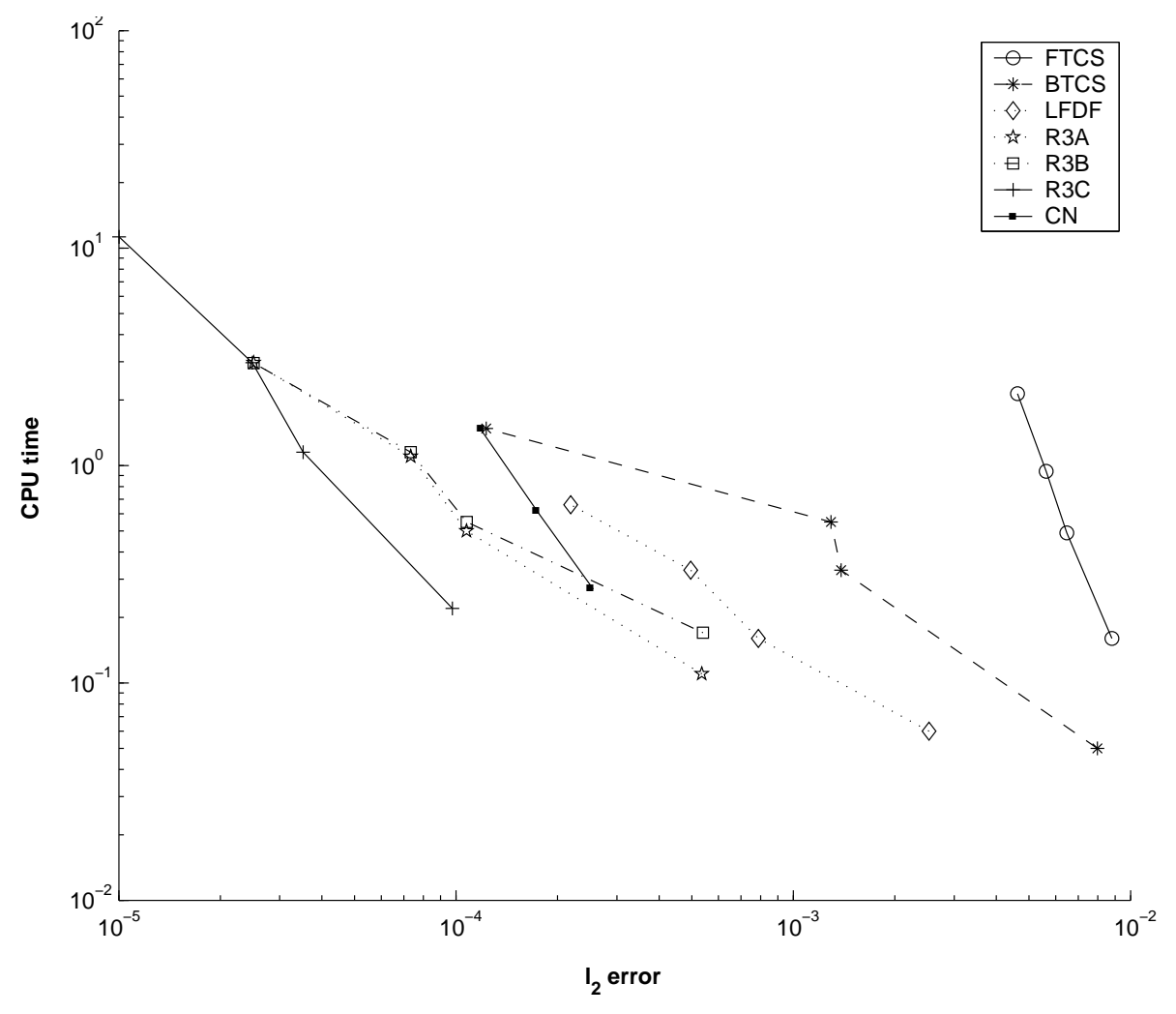

Figure 5: Efficiency: CPU-time vs. $l_{2}$-error.

$$
\sigma_{0}=0.2, \quad \rho=0.1, \quad E=100, \quad T=0.04 .
$$

The solutions at time $t=0.02=1$ year are plotted in Figure 6 for different values of the transaction cost parameter $a$. Figure 7 shows the difference between the Black-Scholes price and the price given by the solution of (4). Since the nonlinear volatility depends on the Gamma $\left(V_{S S}\right)$, the difference is small in regions with small Gamma. The difference is not symmetric. The position of the maximal difference is moving in negative direction in time, relating to the negative sign of the convective term in (7). At one year the maximal difference is at $S=95$. The linear Black-Scholes price is about 9.93 whereas the nonlinear price $(a=0.02)$ is about 12.28 . The nonlinear price is $23.6 \%$ higher than the linear Black-Scholes price.

In financial context the option price sensitivities are known as 'Greeks'. Mathematically, they are the derivatives of the option price with respect to the variables or parameters. The most important ones are the first and second derivatives with respect to the price of the underlying stock, called 'Delta' and 'Gamma', respectively. Since price sensitivities are a distinctive 


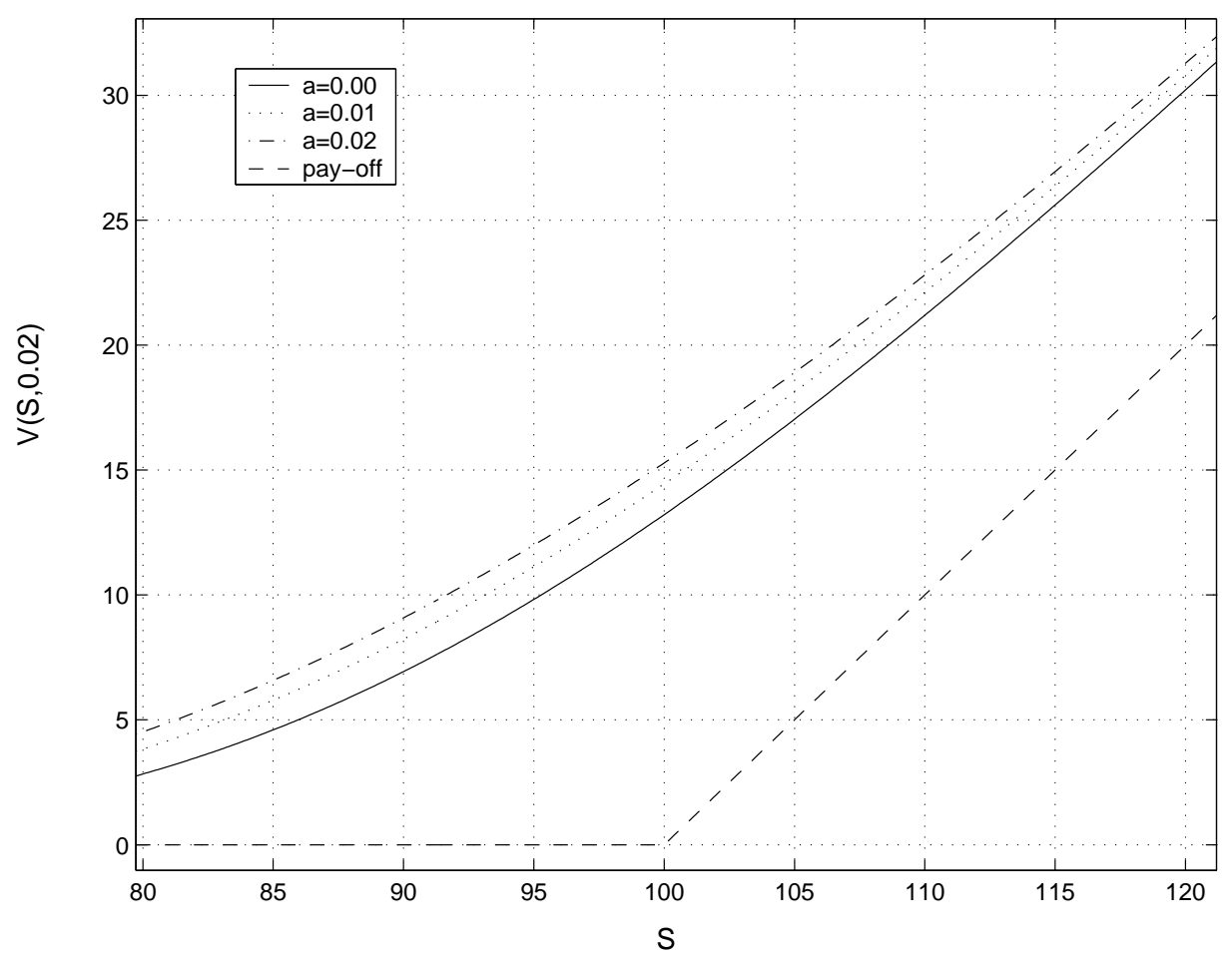

Figure 6: Solution of (4).

measure of risk, growing emphasis on risk management issues has suggested a greater need for their efficient computation.

Figure 8 shows the error of the Greeks of the numerical solution computed using 50 grid points. The following parameters were used in the computation

$$
a=0.02, \quad \sigma_{0}=0.2, \quad \rho=0.1, \quad E=100, \quad T=0.02 .
$$

The Greeks were computed using the standard fourth order central difference approximation of the numerical solutions of (4). We observe that the compact scheme R3C gives the best approximation. The Crank-Nicolson scheme and the compact schemes R3A and R3B also produce acceptable results. The errors of the classical schemes (FTCS, BTCS, LFDF) are up to three times larger than those of the compact schemes. The Leap-Frog Du Fort-Frankel scheme even produces spurious oscillations in the derivatives (not shown). 


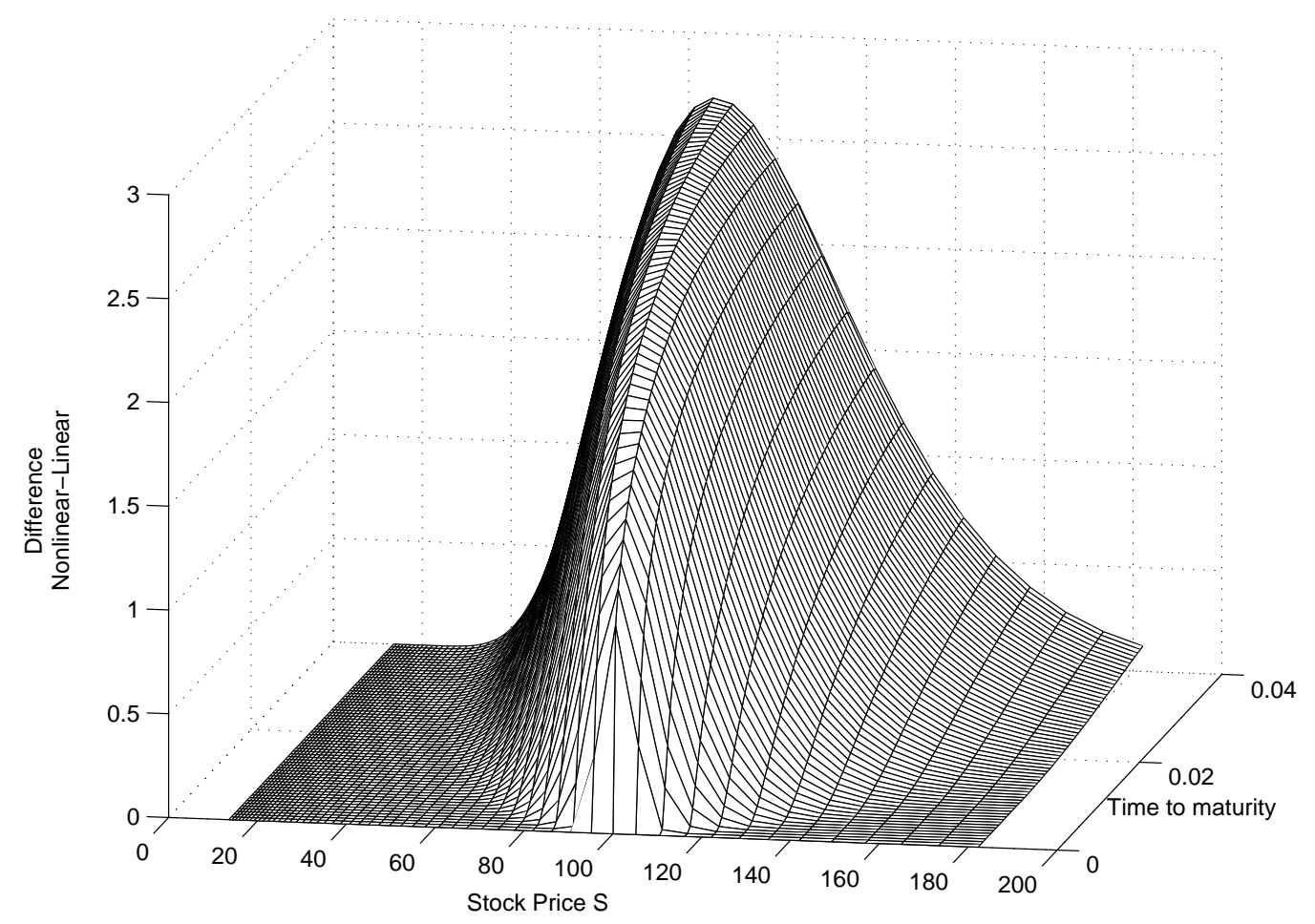

Figure 7: Influence of transaction costs.

\section{Conclusions}

We have derived a new compact scheme R3C generalizing the schemes R3A and R3B derived by Rigal. It turns out that the compact schemes, applied to a semi-implicitly discretized nonlinear Black-Scholes equation, give significantly better results than classical schemes. More precisely,

- they give significantly better accuracy;

- their use is not restricted by strong stability or non-oscillatory conditions; and

- their CPU time is not much larger than that of the classical schemes.

The compact schemes combine good properties (stability, non-oscillations) with a high order of accuracy. The errors in the Greeks Delta and Gamma, computed with the compact scheme R3C, are about one third of the corresponding errors using the BTCS scheme and about half of the errors using the $\mathrm{CN}$ scheme. 

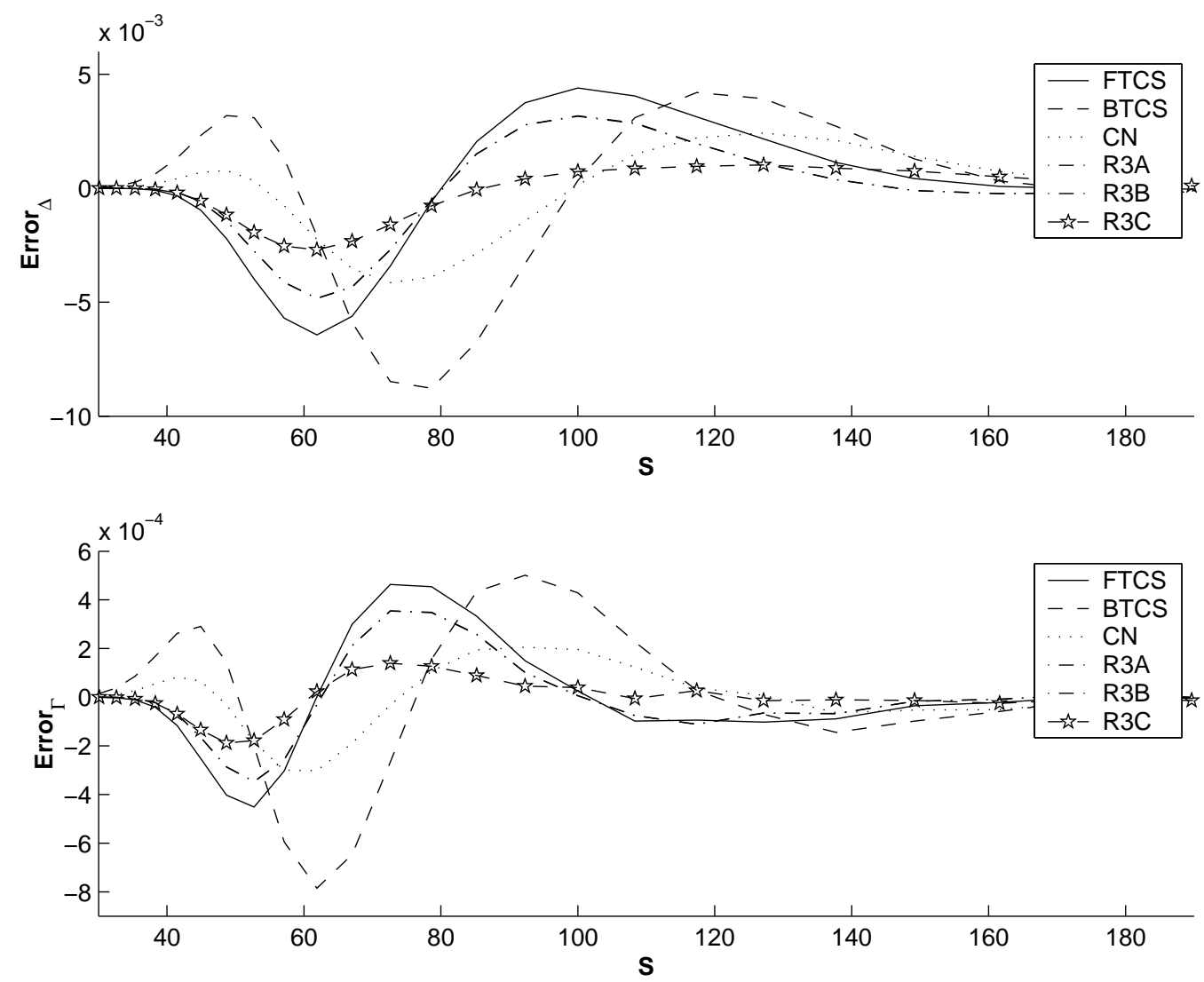

Figure 8: Error in the Greeks Delta (upper figure) and Gamma (lower figure).

These results indicate that compact schemes seem to be an efficient tool in the numerical analysis of option pricing. 


\section{References}

[1] G. Barles, H.M. Soner. Option Pricing with transaction costs and a nonlinear Black-Scholes equation. Finance Stochast. 2, 369-397, 1998. $2,3,4,5$

[2] B. Bensaid, J. Lesne, H. Pagès, J. Scheinkman. Derivative asset pricing with transaction costs. Math. Finance 2, 63-86, 1992. 3

[3] G. Ben-Yu. Generalized stability of discretization and its applications to numerical solutions of nonlinear differential equations. American Mathematical Society. Contemp. Math. 163, 33-54, 1994. 4

[4] A.E. Berger, J.M. Salomon, M. Ciment, S.H. Leventhal, B.C. Weinberg. Generalised OCI Schemes for Boundary Layer Problems. Math. of Comp. 35(151), 695-731, 1980.

[5] F. Black, M. Scholes. The pricing of options and corporate liabilities. J. Polit. Econ. 81, 637-659, 1973. 2

[6] P. Boyle, T. Vorst. Option replication in discrete time with transaction costs. J. Finance 47, 271-293, 1973. 2

[7] G.H. Bruce, D.W. Peaceman, H.H. Rachford, and J.D. Rice. Calculations of Unsteady-State Gas Flow through Porous Media. Trans. Am. Inst. Min. Engrs. (Petrol Div.) 198, 79-92, 1953. 6

[8] G.M. Constantinides, T. Zariphopoulou. Bounds on process of contingent claims in an intertemporal economy with proportional transaction costs and general preferences. Finance Stoch. 3(3), 345-369, 1999. 2

[9] M. Davis, V. Panis, T. Zariphopoulou. European option pricing with transaction fees. SIAM J. Contr. Optim. 31, 470-493, 1993. 2

[10] J. Dewynne, S. Howison, P. Wilmott. Option pricing: mathematical models and computation. Oxford, Financial Press, 1995. 2, 3

[11] P. Forsyth, K. Vetzal and R. Zvan. A finite element approach to the pricing of discrete lookbacks with stochastic volatility. Appl. Math. Finance 6, 87-106, 1999. 3

[12] R. Frey. Perfect option hedging for a large trader. Finance Stochast. 2, 115-141, 1998. 2 
[13] R. Frey. Market illiquidity as a source of model risk in dynamic hedging. In Model Risk, R. Gibson (ed.), RISK Publications, London, 2000. 2

[14] G. Genotte, H. Leland. Market liquidity, hedging and crashes. Amer. Econ. Rev. 80, 999-1021, 1990. 2

[15] S.D. Hodges, A. Neuberger. Optimal replication of contingent claims under transaction costs. Rev. Future Markets 8, 222-239, 1989. 2

[16] R. Jarrow. Market manipulation, bubbles, corners and short squeezes. J. Financial Quant. Anal. 27, 311-336, 1992. 2

[17] P. Kangro, R. Nicolaides. Far field boundary conditions for Black-Scholes equations. SIAM J. Numer. Anal. 38, 1357-1368, 2000. 8

[18] H.B. Keller. Approximation methods for nonlinear problems with application to two-point boundary value problems. Math. Comp. 29, 464-476, 1975. 4

[19] S. Kusuoka. Option replication cost with transaction costs. In Nonlinear and convex analysis in economic theory, Maruyama, Toru (ed.), Proceedings of the T.I. Tech/ K.E.S. conference on nonlinear and convex analysis in economic theory, Keio University, Japan, July 2-4, 1993. Lect. Notes Econ. Math. Syst. 419, 197-202, 1995. 2

[20] Y. Lai and J. Spanier. Applications of Monte Carlo/quasi-Monte Carlo methods in finance: Option pricing. In Monte Carlo/quasi-Monte Carlo Methods, H. Niederreiter (ed.), , Proceedings of a conference held at the Claremont Graduate Univ., CA, USA, June 1998. Springer, Berlin, 2000. 3

[21] D. Lamberton. Error estimates for the binomial approximation of American put options. Ann. Appl. Probab. 8, 206-233, 1998. 3

[22] J. Leitner. Utility maximisation and duality. Preprint Zentrum für Finanzen und Ökonometrie Nr. 00/34, University of Konstanz, Germany, 2000. 2

[23] H. Leland. Option pricing and replication with transaction costs. J. Finance 40, 1283-1301, 1985. 2

[24] R.C. Merton. Theory of rational option pricing. Bell J. Econ. Manag. Sci. 4, 141-183, 1973. 2 
[25] A. Parás, M. Avellaneda. Dynamic hedging portfolios for derivative securities in the presence of large transaction costs. Appl. Math. Finance 1, 165-193, 1994. 2, 3

[26] E. Platen, M. Schweizer. On feedback effects from hedging derivatives. Math. Finance 8, 67-84, 1998. 2

[27] O. Pironneau and F. Hecht. Mesh adaption for the Black and Scholes equations. East-West J. Numer. Math. 8, 25-35, 2000. 3

[28] R.D. Richtmyer, K.W. Morton. Difference Methods for Initial Value Problems. Interscience, New York, 1967. 12

[29] A. Rigal. High Order Difference Schemes for Unsteady One-Dimensional Diffusion-Convection Problems. J. Comp. Phys. 114 (1), 59-76, 1994. 4, 6, 10, 11, 13, 16

[30] A. Rigal. Numerical Analysis of three-time-level Finite Difference Schemes for Unsteady Diffusion-Convection Problems. J. Num. Meth. Engineering 30, 307-330, 1990. 10

[31] P. Schönbucher, P. Wilmott. Hedging in illiquid markets: nonlinear effects. Z. Angew. Math. Mech. 76, Suppl. 3, 81-84, 1996.

[32] P. Schönbucher, P. Wilmott. The feedback effect of hedging in illiquid markets. SIAM J. Appl. Math. 61, 232-272, 2000. 2

[33] K.R. Sircar, G. Papanicolaou. General Black-Scholes models accounting for increased market volatility from hedging strategies. Appl. Math. Finance 5 (1), 45-82, 1998.

[34] H.J. Stetter. Analysis of discretization methods for ordinary differential equations. Springer Tracts in Natural Philosophy. Vol. 23, 1973. 4

[35] J.C. Strikwerda. Finite Difference Schemes and Partial Differential Equations. Wadsworth \& Brooks/Cole, Mathematics series, 1989. 3, 8, 9, 19

[36] R.F. Warming, B.J. Hyett. The modified equation approach to the stability and accuracy analysis of finite-difference methods. J. Comput. Phys. 14, 159-179, 1974. 11

[37] A. Whalley, P. Wilmott. An asymptotic analysis of an optimal hedging model for option pricing with transaction costs. Math. Finance 7, 307324, 1997. 2 